\title{
Free Vibration of Smart Carbon Nanotube Reinforced Composite Skew Panels with Variable Radius of Curvature
}

\author{
A. R. Setoodeh, M. Shojaee, and P. Malekzadeh
}

\begin{abstract}
Free vibration analysis of functionally graded carbon nanotube reinforced composite (FG-CNTRC) skew panels with surface-bonded piezoelectric layers is developed. First-order shear deformation theory is used to model the displacement fields of skew panels with variable radius of curvature. Hamilton's principle and Maxwell's equation are applied to drive the differential governing equations and the related boundary conditions. The transformed differential quadrature (TDQ) method for the case of skew panels with variable radius of curvature is established to discretize the governing partial differential equations. The accuracy and reliability of the proposed method are verified by comparing the results with the existing reference solutions. The effects of CNTs volume fractions, CNTs distributions through the thickness, and various panel geometrical parameters such as the panel thickness, piezoelectric thickness, and skew angle on the natural frequency parameter are demonstrated.
\end{abstract}

Index Terms-Free vibration, smart skew panels, piezoelectric, transformed differential quadrature, carbon nanotubes.

\section{INTRODUCTION}

Shells and panels with complicated geometry have been applied in many areas such as mechanical, civil, and aerospace engineering [1]. In other hand, carbon nanotubes due to superior mechanical and electrical properties is used to reinforce composites [2]. In addition, employing the technology of smart piezoelectric structures which provide the ability to perform self-diagnosis and adaption to the environment change, has become more widespread over the last decade [3]. Practically, singly-curved skew shells embedded in piezoelectric layers with variable radius of curvature as a general geometry of structures such as elliptical, sinusoidal, parabolic and cylindrical panels have many applications in aerospace vehicles, marine vehicles, automotive vehicle and civil structures. Thus, in this research, free vibration of smart functionally graded carbon nanotube reinforced composite (FG-CNRC) singly-curved skew shells with variable radius of curvature is considered. Then, by using transformed differential quadrature (TDQ) method [4]-[6], the discretized form of governing equations are achieved without transferring governing equations to

Manuscript received June 3, 2018; revised August 5, 2018

A. R. Setoodeh and M. Shojaee are with the Department of Mechanical and Aerospace Engineering, Shiraz University of Technology, Shiraz, 71557-313, Iran (e-mail: setoodeh@sutech.ac.ir, m.shojaee@sutech.ac.ir).

P. Malekzadeh is with the Department of Mechanical Engineering, Persian Gulf University, Bushehr, Iran (e-mail: malekzadeh@pgu.ac.ir). computational domain. It is worth noting that the present approach can be further extended for analysis of corrugated skew panel employing the transformed element differential quadrature method.

\section{THE GEOMETRY AND MATERIALS OF THE PANEL}

In Fig. 1, FG-CNTRC skewed panels with variable radius of curvature are shown. As it is obvious from this figure, the panel has a skew angle $\alpha$, subtended angle $\theta_{0}$, length $a$ in the $\xi$-direction, width $b$ in the oblique direction and radius of curvature of the middle surface $R(\eta)$. Also, $h_{c}, h_{p}$ and $h$ denote the core thickness, piezoelectric thickness and total thickness, respectively. A coordinate system with the coordinate variables $\xi, \eta$ and $z$ is used to specify the material points of the variable radius panel in the un-deformed reference configuration.

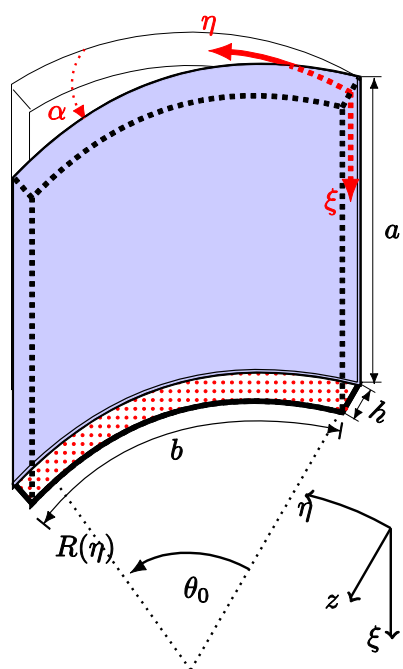

Fig. 1. The geometry of the smart FG-CNTRC skewed cylindrica panels.

The piezoelectric layers are made of homogeneous and isotropic materials, and has a linear elastic behavior. Material properties of core is a matrix reinforced with straight single-walled carbon nanotubes (SWCNTs) with five different distributions of CNTs in the thickness direction which are the uniform distribution (UD), FG-X shape, FG-O shape and FG-V shape distributions (see Fig. 2). The mentioned volume fractions of the CNTs are shown in Fig. 2, respectively.

\section{THE TDQ DiSCRETIZATION ALGORITHM}

By using Hamilton principle and Maxwell's equation, the governing differential equations in terms of displacement 
components are obtained. After that, for the first time, the TDQ method as an efficient and accurate numerical tool is used to study the vibrational behavior of smart FG-CNTRC skewed panels with variable radius of curvature. For this purpose, the transformed weighting coefficients are calculated by using the mapping between the computational and physical domains. The mapping between the physical and computational domains is expressed as follows, $\xi=a \bar{\xi}+\left(R \theta_{0} \tan \alpha\right) \bar{\eta}, \eta=\theta_{0} \bar{\eta}$.

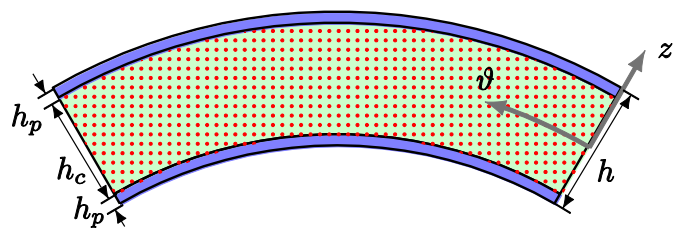

(a) UD

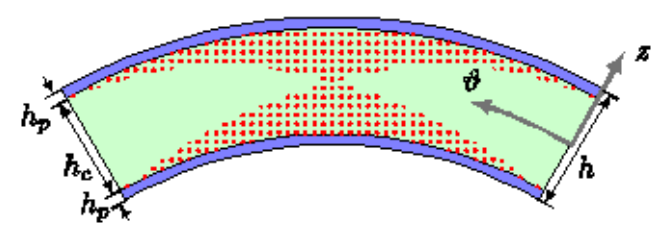

(b) FG-X

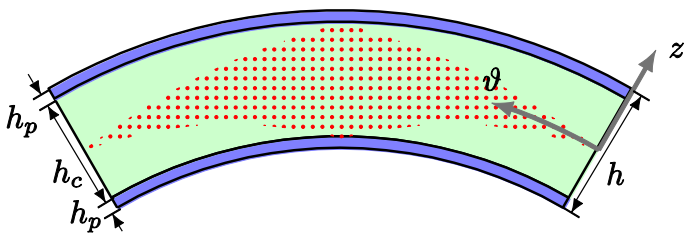

(c) FG-O

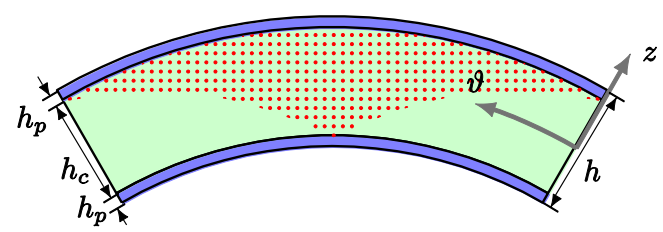

(d) FG-V

Fig. 2. (a)-(d): Different arrangement of CNTs distributions through the panel thickness (a) UD, (b) FG-X, (c) FG-O, (d) FG-V.

Also, the flowchart of the TDQ method and the discretization procedure is illustrated in Fig. 3.

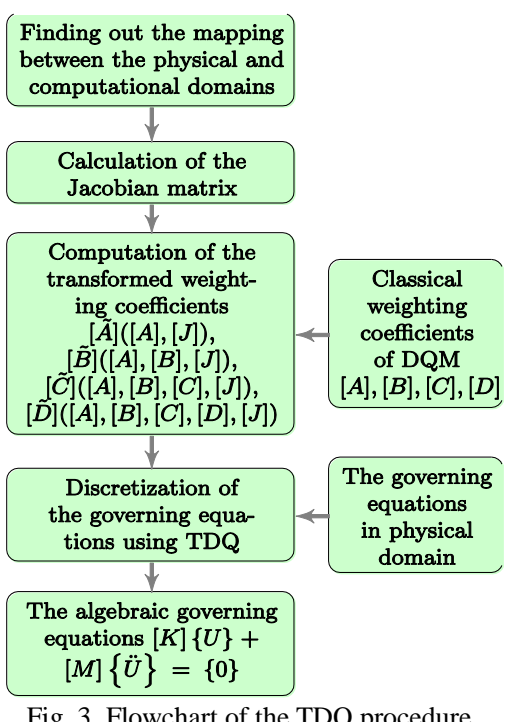

Fig. 3. Flowchart of the TDQ procedure.
In addition, the material properties of the piezoelectric layer, made of lead zirconate titanate (PZT-4), are chosen from [8].

\section{NUMERICAL RESULTS}

Material properties of polymer composite reinforced by single-walled CNTs for three different volume fractions are illustrated in Table I.

TABLE I: MATERIAL PROPERTIES OF POLYMER COMPOSITE REINFORCED BY $(10,10)$ SingLE-WALLED CNTS AT RoOM TEMPERATURE [7]

\begin{tabular}{llllll}
\hline$V_{C N T}^{*}$ & $E_{11}(\mathrm{GPa})$ & $\eta_{1}$ & $E_{22}(\mathrm{GPa})$ & $\eta_{2}$ & $\eta_{3}$ \\
\hline 0.11 & 94.4168 & 0.149 & 2.2037 & 0.934 & 0.934 \\
0.14 & 120.3846 & 0.15 & 2.2977 & 0.941 & 0.941 \\
0.17 & 144.7714 & 0.149 & 3.4939 & 1.381 & 1.381 \\
\hline
\end{tabular}

\section{A. Convergence Study}

Table II demonstrates the convergence patterns of a fully clamped smart FG-CNTRC skew panel with variable radius of curvature. Similarly, as the number of grid points increases from 11 to 29 , a very fast rate of convergence is obtained. Hereafter, we use twenty two grid point $\left(N_{\xi}=N_{\eta}=22\right)$ in each direction to obtain the well-converged, accurate results.

TABLE II: ThE FIRST FIVE NON-DimensionAl FreQUENCY $\left(\lambda_{i}=\omega_{i}\left(a^{2} / h\right) \sqrt{\rho_{m} / E_{m}}\right) \quad$ OF THE FULly ClAMPED FG-CNTR ELLIPTICAL SKew PANEL With PIEZOELECTRIC LAYER $\left(R_{b} / R_{a}=1.5 / 1, b=1\right.$, $\left.a=1, h_{p} / h_{c}=0.1, V_{C N T}^{*}=0.17\right)$

\begin{tabular}{cccccc}
\hline$N_{\xi}$ & $\lambda_{1}$ & $\lambda_{2}$ & $\lambda_{3}$ & $\lambda_{4}$ & $\lambda_{5}$ \\
\hline 11 & 43.8473 & 46.8998 & 52.1412 & 59.7231 & 71.2422 \\
15 & 43.8486 & 46.9155 & 51.6916 & 58.5607 & 67.1680 \\
19 & 43.8482 & 46.9146 & 51.6864 & 58.4883 & 67.1314 \\
21 & 43.8482 & 46.9145 & 51.6862 & 58.4875 & 67.1268 \\
25 & 43.8481 & 46.9144 & 51.6861 & 58.4873 & 67.1268 \\
27 & 43.8481 & 46.9144 & 51.6860 & 58.4873 & 67.1267 \\
29 & 43.8481 & 46.9144 & 51.6860 & 58.4873 & 67.1267 \\
\hline
\end{tabular}

TABLE III: COMPARISON OF FUNDAMENTAL FREQUENCY PARAMETER $\left(\tilde{\lambda}=\omega R \sqrt{\rho / E}\right.$ ) OF HOMOGENEOUS SKEWED CYLINDRICAL PANEL ( $\alpha=45^{\circ}$, $\left.h / R=0.01, v=0.3, k_{s}=5 / 6, E=210 \mathrm{GPa}, \rho=7800 \mathrm{~kg} / \mathrm{m}^{3}\right)$

\begin{tabular}{|c|c|c|c|c|c|c|}
\hline & \multicolumn{3}{|l|}{$\theta_{0}=30^{\circ}$} & \multicolumn{2}{|c|}{$\theta_{0}=60^{\circ}$} & \multirow[b]{2}{*}{$a / R_{a}=3$} \\
\hline & $a / R_{a}=0.5$ & $a / R_{a}=1$ & $a / R_{a}=3$ & $a / R_{a}=0.5$ & $a / R_{a}=1$ & \\
\hline TDQ & 0.5016 & 0.1695 & 0.0293 & 0.5016 & 0.1795 & 0.0396 \\
\hline P-type[9] & 0.505 & 0.170 & 0.030 & 0.509 & 0.181 & 0.040 \\
\hline I-DEAS[9] & 0.500 & 0.169 & 0.029 & 0.484 & 0.177 & 0.039 \\
\hline
\end{tabular}

\section{B. Comparison Study}

As a second example, the present formulation is validated by comparing the obtained non-dimensional frequency parameters of a homogeneous skewed cylindrical panel with those of finite element solution (P-type, I-DEAS) reported by Kandasamy and Singh [9] in Table III. The boundary 
conditions of the panel are clamped at the curved edges and free at the straight sides. For three different values of the length-to-radius ratios and two subtended angles, the results are prepared. As it can be seen, a very good agreement is achieved in all cases under consideration.

\section{Parametric Studies}

After validating the present formulation and the method of solution, some numerical results for the FG-CNTRC skew panel with and without piezoelectric layers are prepared. For brevity, elliptical skew panels with the following geometry, as a general example are considered, hereafter.

Geometry of elliptical skew panel: $b=1 \mathrm{~m}, a=1 \mathrm{~m}, h_{c}=0.1 \mathrm{~m}$, $R_{b} / R_{a}=1.5 / 1$

In Fig. 4, the variation of the first non-dimensional frequency parameters versus the thickness ratio for the smart FG-CNTRC skewed elliptical panels with FG-X distribution of CNTs is depicted. In all cases, it is observed that the frequency parameters increase monotonically as the thickness ratio increases. Also, it can be seen that the increase of piezoelectric thickness has low effect on the non-dimensional natural frequency when the piezoelectric thickness is more than 0.1. In Fig. 5, the influences of skew angles together with CNTs volume fraction on the fundamental frequency parameter of the FG-CNTRC skewed elliptical panels with and without piezoelectric layer are investigated.

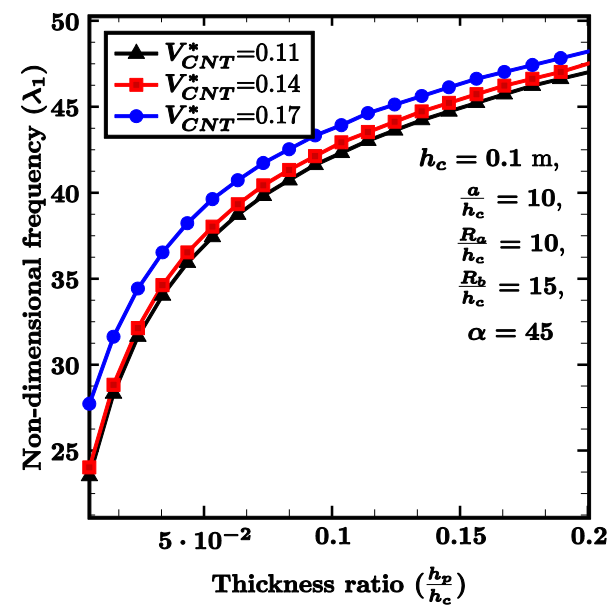

Fig. 4. The variation of first three frequency parameter $\left(\lambda_{i}=\omega_{i}\left(a^{2} / h\right) \sqrt{\rho_{m} / E_{m}}\right)$ versus skew angle for fully clamped FG-CNTRC skewed elliptical panels (FG-X).

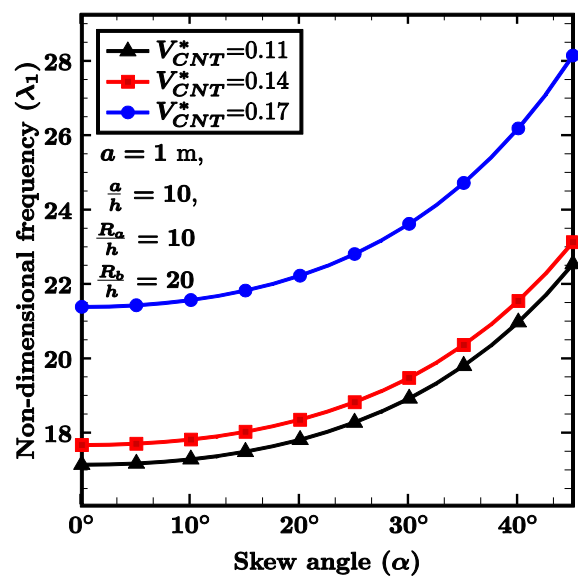

(a) Without piezoelectric layer

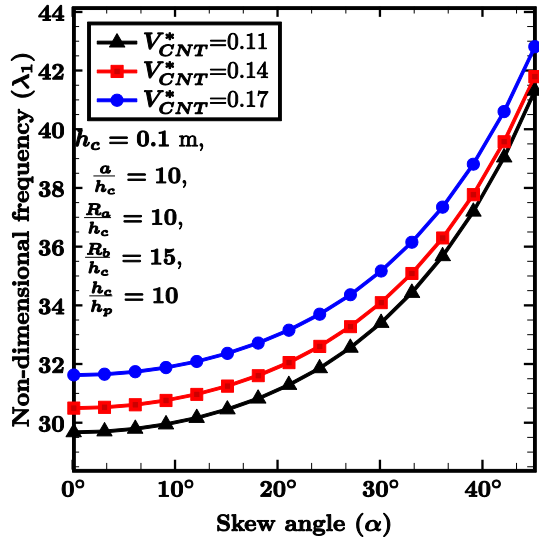

(b) With piezoelectric layers

Figure 5. The variation of fundamental frequency parameter $\left(\lambda_{i}=\omega_{i}\left(a^{2} / h\right) \sqrt{\rho_{m} / E_{m}}\right)$ against the skew angle for fully clamped FG-CNTRC skewed elliptical panels (FG-X).

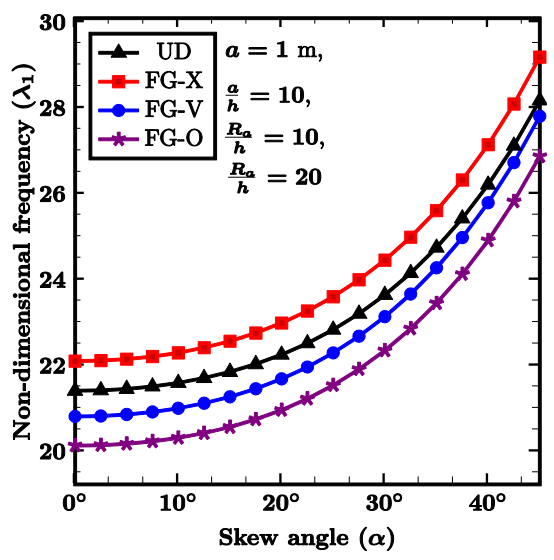

(a) Without piezoelectric layer

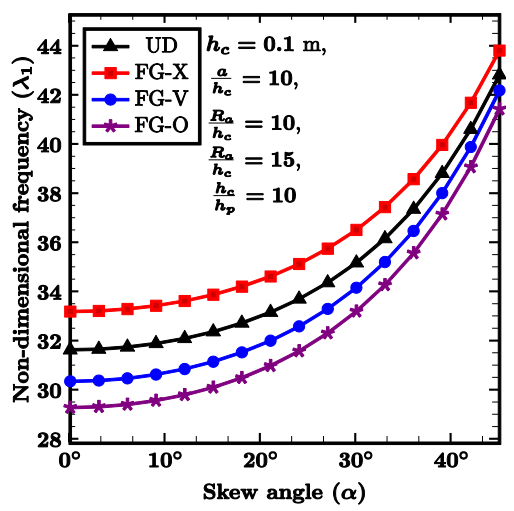

(b) With piezoelectric layer

Figure 6 . The variation of fundamental frequency parameter against skew angle for fully clamped FG-CNTRC skewed elliptical panels with different distribution of CNTs $\left(V_{C N T}^{*}=0.17\right)$.

In those cases, it is seen that the frequency parameters increase significantly as the skew angle increases. In addition, by a slight increase in the CNTs volume fraction, the frequency parameters vary considerably. Fig. 6 shows the impact of the skew angle on the fundamental frequency parameter of the fully clamped FG-CNTRC skewed elliptical panels with and without piezoelectric layer for different distribution of CNTs. One can see that in the skew panel with and without piezoelectric layer, the panels with the FG-X and FG-O distributions of the CNTs have the greatest and lowest 
natural frequencies, respectively.

\section{CONCLUSIONS}

The free vibration behavior of smart FG-CNTRC skew panels with variable radius of curvature was studied. The governing equations were derived based on the FSDT of shells. An extended version of the DQM, called TDQ method was employed to discretize the strong form of the governing equations and the related boundary conditions. As a special case, the FG-CNTRC elliptical skew panels with variable radius of curvature were selected for numerical result. The results show that the skew angle can significantly affect the frequency parameter. Due to the high accuracy of the TDQ method, the presented new solution for FG-CNTRC skew panels with variable radius of curvature can be used as benchmark for future works.

\section{REFERENCES}

[1] H. Altenbach and G. I. Mikhasev, Shell and Membrane Theories in Mechanics and Biology, Cham: Springer International Publishing, 2015 , ch. 1.

[2] K. M. Liew, Z. X. Lei, and L. W. Zhang, "Mechanical analysis of functionally graded carbon nanotube reinforced composites: A review Comp. Struct., vol. 120, pp. 90-97.

[3] H. S. Tzou andJ. P. Zhong, "Adaptive piezoelectric shell structures: theory and experiments," Mech. Syst. Signal Process, vol. 7, pp. 307-319, 1993.

[4] A. R. Setoodeh, and M. Shojaee, "Critical buckling load optimization of functionally graded carbon nanotube-reinforced laminated composite quadrilateral plates," Polymer Compos., 2017.

[5] M. Shojaee, A. R. Setoodeh, and P. Malekzadeh, "Vibration of functionally graded CNTs-reinforced skewed cylindrical panels using a transformed differential quadrature method," Acta Mechanica, pp. $1-21,2017$.

[6] A. R. Setoodeh and M. Shojaee, "Application of TW-DQ method to nonlinear free vibration analysis of FG carbon nanotube-reinforced composite quadrilateral plates," Thin-Walled Struct., vol. 108, pp. $1-11,2016$

[7] H. S. Shen, "Nonlinear bending of functionally graded carbon nanotube-reinforced composite plates in thermal environments," Comp. Struct., vol. 91, pp. 9-19, 2009.

[8] H. Sayyaadi, and M. A. A. Farsangi, "An analytical solution for dynamic behavior of thick doubly curved functionally graded smart panels," Comp. Struct., vol. 107, pp. 88-102, 2014.

[9] S. Kandasamy and A. V. Singh, "Free vibration analysis of skewed open circular cylindrical shells," J. Sound \& Vib., vol. 290, pp. 1100-1118, 2006.

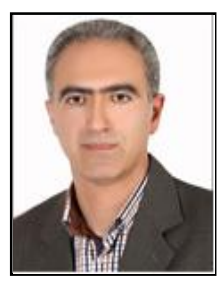

AliReza Setoodeh is a full professor of mechanical engineering in the Department of Mechanical and Aerospace Engineering, Shiraz University of Technology. He obtained his PhD, M.Sc. and B.Sc from Shiraz University. He has published more than 70 papers in the refereed international journals and conferences. His research area of interests are composites, nanocomposites, computational mechanics and mechanical elements design.

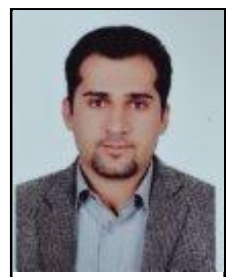

Mohammad Shojaee was born in Fars, Iran on September 19, 1987. He received his bachelor's degree in mechanical engineering in 2009 from Islamic Azad University. Afterwards, he obtained his master's degree in Mechanical Engineering at Persian Gulf University. Currently, he is a PhD student of Mechanical Engineering in Shiraz University of Technology.

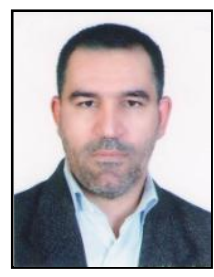

Parviz Malekzadeh is a full professor of mechanica engineering in the Department of Mechanical Engineering, Persian Gulf university. He obtained his $\mathrm{PhD}$, M.Sc. and B.Sc. from Shiraz University. He has published more than 150 papers in the refereed international journals and conferences. His research area of interests are nonlinear vibration, mechanics of composite and nanocomposite materials and computational mechanics. 